\title{
CONSTRUÇÃO DE BASES DE DADOS APLICADAS EM DIFERENTES ATIVIDADES DE ENFERMAGEM*
}

\author{
Magda Rojas Yoshioca, ** \\ Maria Alves Barbosa, *** \\ Marly Theoto Rocha, *** \\ Lisabelle Mariano Rossato, **** \\ Vera Lúcia Maria Regina,+ \\ Fernanda Anqjas Caldas Farias, + \\ Abigail Moura Rodrigues, ++
}

YOSHIOCA, M. R. et al. Construção de base de dados aplicadas em diferentes atividadeo de Enfermagem. Rov.Eac.Enf.USP, v.28, n.1, p. 27-40, abril, 1994.

$O$ presente trabalho mostra a importância da utilizaçāo da informática na enfermagem e revela que é possível criar bases de dados informatizadas, mesmo que o seu autor nāo seja um "expert" no assunto. Descreve bases de dados desenvolvidas no transcorrer da disciplina de informática do Programa de Pós-Graduação da Escola de Enfermagem da Univeraidade de São Paulo.

UNITERMOS: Base de Dados, Informática.

\section{INTRODUÇÃO}

As disciplinas que tratam do assunto Informática e suas aplicações na enfermagem são matérias relativamente novas nos currículos de algumas escolas, a exemplo da Escola de Enfermagem da Universidade de São Paulo (EEUSP), da Faculdade de Enfermagem e Nutrição da Universidade Federal de Mato Grosso e o Departamento de Enfermagem da Escola Paulista de Medicina, que vem oferecendo estas disciplinas nos cursos de graduação e pós-graduaçāo.

Os docentes do curso de pós-graduaçāo da EEUSP sentindo que o profissional de enfermagem nāo pode continuar alheio à essa tecnologia, introduziram a disciplina "Aplicaçōes da Informática na Enfermagem" em seu

\footnotetext{
- Trabalho apresentado a dieciplina Aplicaçbes da Informática na enfermagem do curso de pós-graduaç⿰丿丶万⿱⿰㇒一乂 de Escola de Enfermagen da USP

-. Docente da Faculdade de Enfermagem e Nutriglio da UFMT

En Enfermeira. Docente da Faculdade de Enfermagem e Nutriçóo da UFG

•*. Enfermeira. Prof.Dr. do Departamento ENS da EEUSP

$+\quad$ Enfermeira do Instituto Dante Pezzanese de Cardiologia

++ Docente do Departamento de Enfermagem da UFRN
} 
programa, com a finalidade de instrumentalizar o alunoiprofissional para as atividades de pesquisa, ensino e assisténcia.

Essa disciplina foi oferecida pela primeira vez em 1992 aos alunos de pós-graduaçáo em nivel de mestrado e doutorado que, reconhecendo a importância da informática como ferramenta de trabalho, decidiram lançar māo dessa tecnologia e construir algumas bases de dados de utilizaçāo em seus campos de trabalho e/ou pesquisa.

Antes de se fazer a descrição das bases de dados, é necessário contextualizar a informática no campo da saúde, especialmente na enfermagem.

No Brasil a aplicação da informática intensificou-se na área de saúde em torno de 1975, quando era empregada em laboratórios, devido a facilidade e rapidez com que o computador fornece resultados manuseando grandes volumes de informaçōes 4 .

Nas duas últimas décadas houve notável crescimento da aplicação dos recursos de informática na área de saúde, como pode ser constatado através dos trabalhos apresentados em congressos de informática ${ }^{19}$.

No I CONGRESSO BRASILEIRO DE INFORMÁTICA HOSPITALAR realizado em São Paulo (1991) foram apresentados diversos softwares aplicáveis ao setor saúde. Nesta oportunidade, foram divulgados trabalhos que mostravam, inclusive, aplicativos criados para uso exclusivo da enfermagem. Os trabalhos de Mc ALINDON et al ${ }^{11}$, GUIMARÄES et al ${ }^{6}$, entre outros mostram o desenvolvimento de software destinados às diversas atividades realizadas pelo enfermeiro.

Nesse mesmo evento foi enfatizada a importância da informática para a enfermagem, que tem por objetivo aumentar: a produtividade, o tempo de contato do enfermeiro com o cliente à beira do leito, o número de informaçōes precisas e o acesso da equipe a todas as informaçōes.

YOSHIOCA ${ }^{21}$ ao concluir seu trabalho sobre "Informática: nova ferramenta do trabalho da enfermagem "menciona que a informática contribuirá para que haja "maior disponibilidade de tempo para desenvolvermos as relaçōes humanas junto à equipe, aos alunos e a clientela".

GUIMARĀES et al ${ }^{6}$ ao elaborarem o software de prescrição de enfermagem para uso na sala de recuperaçāo pós-anestésica enfatizaram, também, a disponibilidade maior de tempo do enfermeiro junto ao cliente, dizendo ainda que "a informática permite o desenvolvimento de assistência técnica e humanizada".

Alguns trabalhos apontam a importância da informática como ferramenta de trabalho ${ }^{2,7,9,18,21}$, outros mostram a aplicação em áreas específicas como ambulatório especializado ${ }^{20}$, prescriçāo de enfermagem ${ }^{6}$, uso de terminal à beira do leito ${ }^{2}$, avaliação docente ${ }^{5}$, relatório de enfermagem ${ }^{9}$, educaçāo continuada ${ }^{17}$, estabelecimento da relação custo e tempo no assistir ${ }^{18}$ e outros, ainda, descrevem a criação de bancos de dados e suas especificidades $8,10,13,14,16,20$

Mais recentemente, sistemas que utilizam a inteligência artificial têm sido criados para auxiliar a enfermagem em suas atividades. BLOOM et al ${ }^{3}$, desenvolveram um sistema especialista com a finalidade de ajudar o profis- 
programa, com a finalidade de instrumentalizar o alunoiprofissional para as atividades de pesquisa, ensino e assisténcia.

Essa disciplina foi oferecida pela primeira vez em 1992 aos alunos de pós-graduaçáo em nivel de mestrado e doutorado que, reconhecendo a importância da informática como ferramenta de trabalho, decidiram lançar māo dessa tecnologia e construir algumas bases de dados de utilizaçāo em seus campos de trabalho e/ou pesquisa.

Antes de se fazer a descrição das bases de dados, é necessário contextualizar a informática no campo da saúde, especialmente na enfermagem.

No Brasil a aplicação da informática intensificou-se na área de saúde em torno de 1975, quando era empregada em laboratórios, devido a facilidade e rapidez com que o computador fornece resultados manuseando grandes volumes de informaçōes 4 .

Nas duas últimas décadas houve notável crescimento da aplicação dos recursos de informática na área de saúde, como pode ser constatado através dos trabalhos apresentados em congressos de informática ${ }^{19}$.

No I CONGRESSO BRASILEIRO DE INFORMÁTICA HOSPITALAR realizado em São Paulo (1991) foram apresentados diversos softwares aplicáveis ao setor saúde. Nesta oportunidade, foram divulgados trabalhos que mostravam, inclusive, aplicativos criados para uso exclusivo da enfermagem. Os trabalhos de Mc ALINDON et al ${ }^{11}$, GUIMARÄES et al ${ }^{6}$, entre outros mostram o desenvolvimento de software destinados às diversas atividades realizadas pelo enfermeiro.

Nesse mesmo evento foi enfatizada a importância da informática para a enfermagem, que tem por objetivo aumentar: a produtividade, o tempo de contato do enfermeiro com o cliente à beira do leito, o número de informaçōes precisas e o acesso da equipe a todas as informaçōes.

YOSHIOCA ${ }^{21}$ ao concluir seu trabalho sobre "Informática: nova ferramenta do trabalho da enfermagem "menciona que a informática contribuirá para que haja "maior disponibilidade de tempo para desenvolvermos as relaçōes humanas junto à equipe, aos alunos e a clientela".

GUIMARĀES et al ${ }^{6}$ ao elaborarem o software de prescrição de enfermagem para uso na sala de recuperaçāo pós-anestésica enfatizaram, também, a disponibilidade maior de tempo do enfermeiro junto ao cliente, dizendo ainda que "a informática permite o desenvolvimento de assistência técnica e humanizada".

Alguns trabalhos apontam a importância da informática como ferramenta de trabalho ${ }^{2,7,9,18,21}$, outros mostram a aplicação em áreas específicas como ambulatório especializado ${ }^{20}$, prescriçāo de enfermagem ${ }^{6}$, uso de terminal à beira do leito ${ }^{2}$, avaliação docente ${ }^{5}$, relatório de enfermagem ${ }^{9}$, educaçāo continuada ${ }^{17}$, estabelecimento da relação custo e tempo no assistir ${ }^{18}$ e outros, ainda, descrevem a criação de bancos de dados e suas especificidades $8,10,13,14,16,20$

Mais recentemente, sistemas que utilizam a inteligência artificial têm sido criados para auxiliar a enfermagem em suas atividades. BLOOM et al ${ }^{3}$, desenvolveram um sistema especialista com a finalidade de ajudar o profis- 
sional a fazer diagnóstico e elaborar o plano de cuidados. No Brasil MARIN 12 está desenvolvendo um protótipo de sistema especialista em assistência pré-natal no atendimento de gestante normal.

Quanto à base de dados, BOHM et al ${ }^{4}$ afirmam que é "uma coleção integrada de dados organizados em arquivos", ou seja, é uma forma estruturada de armazenar dados/informaçōes, que podem ser analisados posteriormente, em conjunto.

A base de dados, independentemente do tipo de aplicação a que se destina, deve possuir métodos gerais que se encarregam das operaçōes básicas de estruturaçāo, acesso e manuseio de informaçōes. Para exercer suas funçōes adequadamente necessita ser eficiente, nāo redundante, consistente e flexivel 4. Baseado nisso, foram desenvolvidos softwares modernos que permitem ao usuário, especialista ou nảo, criar e manusear banco de dados com maior autonomia.

A construçāo de base de dados deve estar pautada no tripé: o quê, como e para que coletar os dados. O conjunto de informaçōes deve estar organizado de forma a facilitar o dia-a-dia do profissional quer nas suas atividades administrativas, assistenciais, educativas e de pesquisa ${ }^{21}$.

Existem atualmente inúmeros softwares e pacotes de programas que sāo um conjunto de informaçōes organizadas de tal maneira que permitam ao usuário sem conhecimento de informática, seguir uma seqüência de passos e prosseguir o trabalho. Após apreender os elementos básicos de manuseio do microcomputador e de alguns softwares, o usuário é capaz de realizar seu trabalho de digitação de texto, elaboração de gráficos e tabelas, ou gerar banco de dados.

Existem algumas bases de dados informatizadas de referencias bibliográficas na área da saúde, como a LILACS (Literatura Latinoamericana en Ciencia de la Salud), CINAHL (Cumulative Index to Nursing and Allied Health Literature), MEDLINE (Medical Analysis and Revieval System OnLine), DEDALUS (Banco de Dados Bibliográficos da Universidade de São Paulo) e o Banco de Dados de Enfermagem de TEIXEIRA 1,14,19,21.

\section{OBJETIVO}

O objetivo do presente estudo é descrever bases de dados desenvolvidas por enfermeiros, utilizando o software Kards (C).

\section{METODOLOGLA}

Este é um estudo descritivo sobre bases de dados aplicáveis às diversas atividades de enfermagem, que foram construídas por enfermeiros não especialista em informática. 
Dentre os diversos software existentes optou-se pelo Kards (C), cujas características permitem, entre outros, armazenar e manusear informaçōes não categorizadas - textos, realizar cálculos e estatísticas.

Antes de descrever a estrutura de três bases de dados: AISM (dados da clientela de um programa de atendimento à saúde da mulher), EDUC (contém informações sobre funcionários de enfermagem de um hospital) e BIBL (base de dados sobre referências bibliográficas), faz-se necessário discorrer brevemente sobre o que é o software utilizado na construção das mesmas.

\section{O SOFTWARE KARDS (C)}

O software KARDS (C) é um programa gerenciador de bases de dados para computador do tipo $\mathrm{PC}$, requerendo configuração mínima de $256 \mathrm{~Kb}$ de memória e uma unidade de disco rígido. É um sistema integrado de arquivo que mimetiza um fichário de aço para escritório, com gavetas onde sāo guardadas pastas e nestas são arquivadas as fichas ${ }^{15}$. Pode conter até 600 gavetas ordenadas sequencialmente por data, número ou letra, ou ordenadas nāo sequencialmente, por assuntos. Cada gaveta comporta até 150 pastas e cada pasta acomoda até 100 fichas diferentes. Cada ficha pode conter até 20 itens ou 1.000 caracteres.

Dos 9 (nove) módulos disponíveis no KARDS (C) (FICHA, BUSCA, IMPRIME, IMPRESSO, UTIL, MANUTEN, KARDPLAN, KARDGRAF, GERAR) foi utilizado para a construção da estrutura das bases de dados o módulo GERAR ${ }^{15}$.

Para melhor compreensāo da estrutura dos bancos de dados criados com - software Kards (C) é necessário definir alguns códigos utilizados neste trabalho.

$O$ código !IND colocado à frente de alguns itens como exemplo, item da ficha AISMO1.FIC, EDUCO1.FIC E BIBLO1.FIC define os respectivos itens como índices para localização das pastas que contém todas as informações sobre seus "donos" 16 .

O código !COD C encontrado em determinadas fichas, por exemplo itens 3,5 , e 8 de ISMO5.FIC e item 8 de EDUCO1.FIC, facilita o preenchimento do item, evita perda de tempo e erros de digitação do usuário. Para a utilização desse código é necessário construir tabelas com as informaçöes que os itens devem conter ${ }^{15}$. A tabela deve ser elaborada com editor de texto e pode ser atualizada sempre que necessária. Para que o KARDS (C) faça a leitura da tabela o nome do arquivo deve conter o prefixo $\mathrm{CR}$, o nome do fichário (contendo 4 letras), o número da ficha (dois dígitos), e o sufixo.DOC, que é a extensão do nome do arquivo. Exemplificando, temos o arquivo CRAISMO5.DOC referente a tabela da ficha 05 da base de dados denominada AISM (Figura 4).

$\mathrm{O}$ código !TEX empregado nas fichas AISM13.FIC, EDUCA04.FIC E BIBLO2.FIC indica que esta é uma ficha de texto, onde o usuário pode escrever livremente. Esta ficha pode acomodar até 1.000 caracteres ${ }^{15}$. 
$O$ código !CAL realiza cálculos segundo as informaçōes fornecidas. No item 5 da ficha AISMO2.FIC é solicitado o cálculo de renda per capita, o qual se obtém através da divisào rendimento familiar (item 3 ) pelo número de pessoas da família (item 4) ${ }^{15}$.

A delimitação de colunas pode ser realizada utilizando-se o código !TAB, como aparece na ficha EDUCO3.FIC (Figura 8).

\section{BASE DE DADOS AISM}

Os pesquisadores que atuam na área de saúde da mulher necessitam coletar elevado número de informaçōes, para estabelecer o perfil epidemiológico da clientela atendida. Por esse motivo, decidiu-se construir a base de dados AISM que servirá para nortear as açỏes que devem estar contidas em programa de atendimento à Saúde da Mulher.

O banco de dados AISM está composto por 14 fíchas (AISMO1.FIC à AISM14.FIC) e 12 tabelas (CRAISMO1.DOC a CRAISM12.DOC)

$\mathrm{O}$ !COD $\mathrm{C}$, utilizado em várias fichas, aliado às tabelas além de preencher automaticamente os itens correspondentes, faz também a estatística do evento.

A base de dados AISM está alojada em 3 (três) gavetas, dentro das quais seräo acomodadas as pastas das usuárias do serviço de saúde, em ordem alfabética: de $\mathrm{A}$ à $\mathrm{H}(\mathrm{AH})$, de $\mathrm{G}$ à $\mathrm{O}(\mathrm{GO})$ e de $\mathrm{P}$ à $\mathrm{Z}(\mathrm{PZ})$.

Para se ter idéia do teor das informaçóes que constam da base de dados AISM, apresenta-se a seguir a relaçāo das fichas com seus tópicos.

AISMO1.FIC - contém os dados de identificaçāo pessoal (Figura 1). Para ajudar o preenchimento desta ficha dispöe-se do arquivo CRAISMO1.DOC que contém itens relativos ao grau de instrução.

Os dados sociais e de subsistência encontram-se na ficha AISMO2.FIC (Figura 2). $\mathrm{O}$ arquivo CRAISMO2.DOC contém a lista para facilitar o enquadramento da renda per capita.

As informaçōes referentes aos antecedentes pessoais patológicos, estão na ficha AISMO3.FIC. O arquivo CRAISMO3.DOC traz a lista de problemas/patologias mais comuns. O sistema permite a inclusão de outras patologias menos freqüentes.

Os dados referentes a sexualidade estão expressos na ficha AISMO4.FIC. A faixa etária da ocorrência dos aspectos relativos à sexualidade encontram-se no arquivo CRAISMO4.DOC.

Os itens referentes à ginecologia, prevençāo do câncer ginecológico e auto exame da mama encontram-se na ficha AISM05.FIC (Figura 3) e as informaçōes para auxiliar o seu preenchimento estão no arquivo CRAISMO5.DOC (Figura 4).

As informaçóes sobre antecedentes obstétricos estão relacionadas nas fichas AISMO6.FIC (gravidez), AISMO7.FIC (pré-natal), AISMO8.FIC (parto), AISMO9.FIC (puerpério). Estas fichas possuem tabelas que auxiliam o 
preenchimento (CRAISMO6.DOC, CRAISMO7.DOC, CRAISMO8.DOC, CRAISMO9.DOC).

Os dados sobre as condiçōes da criança ao nascer constam da ficha AISM10.FIC, a qual utiliza a tabela CRASIM10.DOC.

As informaçōes sobre planejamento familiar e anticoncepcionais estāo expressas nas fichas AISM11.FIC, AISM12.FIC, AISM13.FIC E AISM14.FIC. O preenchimento das duas primeiras fichas é facilitado quando se usam as tabelas CRAISM11.DOC e CRAISM12.DOC

\section{FIGURAS}

FIGURA 1:

\begin{tabular}{|c|c|c|}
\hline \multicolumn{3}{|l|}{ AISMO1 } \\
\hline \multicolumn{3}{|c|}{$\begin{array}{l}\text { DEFINIÇAO DE FICHA } \\
\text { Item a cocolher: } \\
\text { Eocolhe o item ou use as funçoes }\end{array}$} \\
\hline \multicolumn{3}{|c|}{ IDENTIFICAÇĀO } \\
\hline 1 - NOME: & !IND & \\
\hline 2 - IDADE: & $\begin{array}{l}3 \text { - ESTADO CIVIL } \\
\text { (S/C/N/AO): }\end{array}$ & 4. $\operatorname{COR}(\mathrm{A} / \mathrm{B} / \mathrm{P} / \mathrm{N})$ : \\
\hline \multicolumn{2}{|c|}{5 - GRAU DE INSTRUÇĀO: } & 101;20 !COD C \\
\hline \multicolumn{3}{|c|}{ ENDEREÇO } \\
\hline $\begin{array}{l}\text { 6-RUA: } \\
\text { 9- CEP/CII }\end{array}$ & 7 - BAIRRO: & $\begin{array}{l}\text { 8-FONE: } \\
\text { 10-ESTADO: }\end{array}$ \\
\hline
\end{tabular}

\begin{tabular}{|c|c|c|c|c|}
\hline F1-Inicia & F2.Rinte & F3Aqum & F-10mu & FS-Progreme \\
\hline Fa - Gence & F7.Pun & Fo - Rarinosto & Fo-Areocit & F10-Linte \\
\hline NLT T = Termine pave & ALTD =DOS & ALTH $=$ Apude & Exc = Funpows & \\
\hline
\end{tabular}

\section{FIGURA 2:}

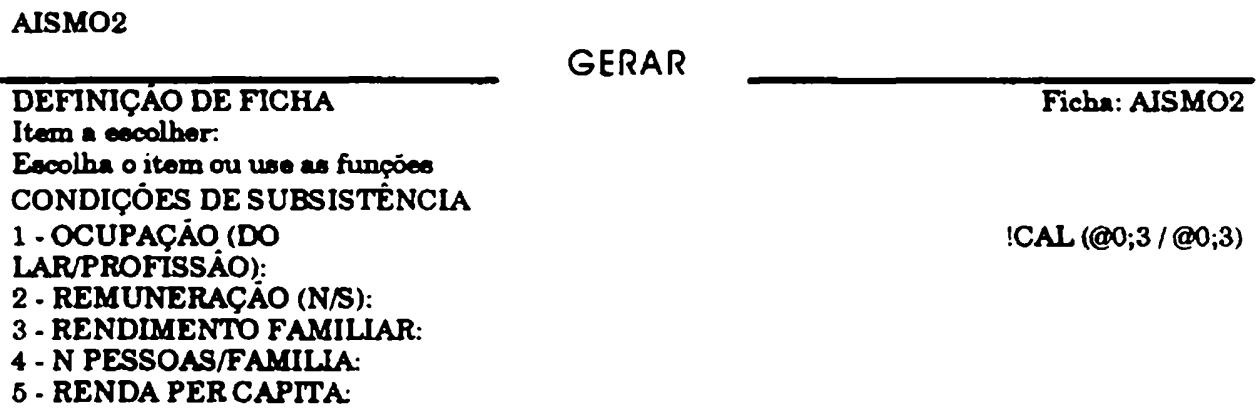

\begin{tabular}{|c|c|c|c|c|}
\hline Fi-Inich & F2. Arman & 7-37nqu & FA- Thenu & F5.Prognem \\
\hline Fo - Gencen & F7. Paxt & Fo - Regirtro & Fo - Ascocis & F10-Linhe \\
\hline NTT = Termina peune & ALT $=$ DOS & ALTH $=$ Auda & Esc $=$ Funpore & \\
\hline
\end{tabular}


FIGURA 3:

AISMO5

DEFINIÇẢO DE FICHA

Fichn: AISMO2

Item a escolher:

Escolhn o item ou use as funçōes

ANTECEDENTES GINECOLOGICOS

1 - CORRIMENTO (S/N):

$01 ; 20 !$ COD C

2 - EXAME PREV Ca GIN (S/N):

3 - ULTIMO EXAME (M/TM-1A/1A/1A-2A/2A):

4 - AUTO EXAME MAMA (S/N):

/01;20 !COD C

5 - MOTIVO NÁO (NST/NSE/NAI):

6 - OUTRO:

7 - GOSTARIA APRENDER

GERAR

(S/N/NS):

8 - CIRUR GIN (N/S):

9 - OUTRA:

10 - CONHECE FUC CORPO (S/N):

11 - GOSTARLA APRENDER (S/N/NS):

\begin{tabular}{|c|c|c|c|c|}
\hline F1-Inicia & F2-Revies & F3 Arqum & F4. Menu & F5 - Programa \\
\hline Fo - Gevero & $F \cdot P=$ & FB - Ropinto & PO-Asecci & F10. Linha \\
\hline ALT T = Termina pace & $A L T D=D O S$ & ALTH $=$ Ahdo & Exc = Fungoes & \\
\hline
\end{tabular}

\title{
FIGURA 4:
}

\author{
CRAISMO5 \\ 01 - 6 MESES \\ 02 - 6 MESES - 1 ANO \\ 03 - 1 - 2 ANOS \\ $04-2$ ANOS \\ 05 - NÁO SABE TÉCNICA \\ 06 - NÁO SABIA QUE EXISTIA \\ 07 - NÁO ACHA IMPORTANTE \\ 08 - OUTRO \\ 09 - COLPOTOMLA \\ 10 - CONIZACÁO \\ 11 - EXERESE CISTO/TU OVARIO \\ 12 - EXERESE CISTO/TU UTERINO \\ 13 - EXERESE CISTOITU VAGINAL \\ 14 - EXERECE CISTOTTU VULVAR \\ 15 - HISTERECTOMIA \\ 16 - SALPINGECTOMIA \\ 17 - MIOMECTOMLA \\ 18 - OOFORECTOMIA \\ 19 - PAN HISTERECTOMLA \\ 20 - PERINEOPLASTIA \\ 21 - OUTRO - QUAL?
}


DEFINIÇẢO DE FICHA

Item a escolher:

Escolha o item ou use as funçoes

OPINLĀO SOBRE PF

1-: !TEX

2-:

3-:

4-:

5-:

6-:

\begin{tabular}{|c|c|c|c|c|}
\hline F1 - Inicia & F2-Aevisa & F3 Arqunva & F4-Menu & F5 - Programa \\
\hline F6. Gaveto & F7 - Pata & FB - Regetro & F9 - Associs & F10-Linha \\
\hline ALT T = Termore pava & ALTD $=$ DOS & ALT $H=$ ANud: & Eec $=$ Fungobes & \\
\hline
\end{tabular}

\section{BASE DE DADOS EDUC}

As enfermeiras que atuam em Educaçāo Continuada, de maneira geral, têm sentido necessidade de organizarem as informaçōes sobre os funcionários sob sua responsabilidade. Diante disso, as autoras elaboraram o prontuário funcional informatizado chamado EDUC contido em 4 gavetas, dentro das quais serāo colocadas as pastas dos funcionários de enfermagem em ordem alfabética, assim distribuídas: de $A$ à $G(A G)$, de $H$ à $L(H L)$, de $M$ à $P$ (MP) e de $Q$ à $Z(Q Z)$.

A pasta do funcionário é composta de 4 (quatro) fichas a saber: EDUCO1.FIC, EDUCO2.FIC, EDUCO3.FIC e EDUCO4.FIC.

Os itens da ficha EDUCO1.FIC contém dados de identificaçāo do funcionário (Figura 6).

$\mathrm{Na}$ ficha EDUCO2.FIC estão contidos os itens sobre o endereçamento do funcionário, para localizá-lo rapidamente sempre que se fizer necessário (Figura 7).

A ficha EUCO3.FIC, em forma de planilha, permite acompanhar a movimentação do funcionário nas diversas unidades do hospital e turnos (Figura 8).

O desempenho do funcionário, suas avaliaçōes, promoçōes, entre outros, estäo descritos, na forma de texto livre, na fixa EDUCO4.FIC (Figura 9). 


\section{FIGURA 6}

EDUCO1.FIC

DEFINIÇÄO DE FICHA

Ficha: EDUCO1

Item a escolher:

Escolba o item ou use as funçòes

IDENTIFICAÇĀO

1 - NOME:

!IND

2 - SEXO:

3 - ESTADOCIVIL:

4 - DATA NASC.:

5 - GRAU DE INSTRUÇÁO:

6 - VEÍCULO (AS):

7 - CARGO/FUNÇȦO:

8 - CATEGORIA:

101;20 !COD C

9 - DATA ADMISSẢO: 10 - MOTIVO (A/S):

\begin{tabular}{|c|c|c|c|c|}
\hline F1-inicia & F2. Alomat & F3 Arquive & F4. Manu & F5 - Programa \\
\hline Fo. Genots & F・Pasta & F8 - Regintro & F9. Aseocin & F10. Lnhe \\
\hline ALT $T=$ Termina pacea & $A L T D=D O S$ & ALTH $=$ ANude & Esc $=$ Funcoses & \\
\hline
\end{tabular}

\section{FIGURA 7}

EDUCO2.FIC

GERAR

DEFINIÇĀO DE FICHA

Ficha: EDUCO2

Item a escolher:

Escollha o item ou use as funcoies

ENDEREÇO

1. RUAAV:

2 - BAIRRO:

3 - CEP:

4. CIDADE:

5 - TELEFONE:

\begin{tabular}{|c|c|c|c|c|}
\hline F1-Inicin & F2-Revien & F3 Arquiva & F4-Menu & F5.Programa \\
\hline Fo. Gevets & F - Pata & F8 - Rogisto & F8 - Aseocin & F10 - Linho \\
\hline ALT $T=$ Termina pave & ALTO $=\infty O S$ & ALTH = Ainde & Esc $=$ Funcoes & \\
\hline
\end{tabular}




\section{FIGURA 8}

EDUCO3.FIC

DEFINIÇĀO DE FICHA

GERAR

Item a escolher:

Escolba o item ou use as funçóes

.

DATA! !-UNIDADE

Ficha: EDUCO3

1-: $\quad$ !TAB

2-: $\quad$ !TAB

3-: $\quad$ ITAB

4-: $\quad$ !TAB

5-: $\quad$ !TAB

\begin{tabular}{|c|c|c|c|c|}
\hline Fi - Incia & F2-Reva & F3 Arquiva & FA.Many & F5-Programa \\
\hline Fo-Gama & F7 - Panta & FB - Aegatro & F9. Assocs & F10-Linna \\
\hline ALT T = Tormina peuse & $A L T D=D O S$ & $A \perp T H=$ Ayude & Eac = Funçbes & \\
\hline
\end{tabular}

\section{FIGURA 9}

\section{EDUCO4.FIC}

DEFINIÇÁO DE FICHA

GERAR

Item a escolber:

Escolha o item ou use as funçóes

DESEMPENHO (AVALIAÇĀO)

: !TEX

\begin{tabular}{|c|c|c|c|c|}
\hline F1-Inicin & F2-Amvias & F3 Arquive & F4-Menu & F5 - Programe \\
\hline F6-Gaveta & F7. Pacta & Fo - Regutro & Fg-Aleocia & F10. Linina \\
\hline ALT T = Termina pause & $A L T D=D O S$ & $A L T H=$ Aude & Ese $=$ Funçoes & \\
\hline
\end{tabular}

\section{BASE DE DADOS BIBL}

A utilizaçāo de publicaçōes de artigos científicos é freqüentemente requerida em atividades tais como ensino, pesquisa, extensảo ou mesmo para leitura diária. Com a finalidade de facilitar o acesso a essas informaçōes criou-se a base de dados BIBL.

A principal vantagem de se criar uma "biblioteca particular" (BIBL) é o fato de seus autores conhecerem a estrutura e o conteúdo da mesma, pois eles selecionam os trabalhos que farão parte da base de dados, criam a lista dos descritores de assunto e estabelecem as regras para a classificaçāo das referências 13,14 .

A base de dados BIBL foi organizada em duas gavetas. A gaveta $A L$ contém pastas de $A$ à $L$, a gaveta $M Z$ acomodam as pastas de $M$ à $Z$, estando 
portanto em ordem alfabética. Cada pasta contém 2 (duas) fichas, a ficha BIBLO1.FIC e BIBLO2.FIC.

Os itens da ficha BIBLO1.FIC caracterizam a referència bibliográfica propriamente dita, além de conter palavras-chaves, o que facilita o acesso ao assunto pesquisado (Figura 10).

A ficha BIBLO2.FIC é uma ficha texto que possibilita ao usuário digitar o resumo da referència bibliográfica (Figura 11).

\section{FIGURA 10}

\section{BIBLO1.FIC}

DEFINIÇÁO DE FICHA

GERAR

Item a escolher:

Escolha o item ou use as funçóes

REFERENCLAS

1 - AUTOR:

3. TITULO:

4 - PERIODICO:

5 - LIVRO:

8 - VOLUME:

9 - NÚMERO:

10 - PAGINA:

11- ANO:

12 - PALAVRAS-CHAVES:

\begin{tabular}{|c|c|c|c|c|}
\hline FI.Inich & F2. Anovina & F3 Aquitve & Fa-Menu & F5 - Programs \\
\hline Fo-Gente & F7.Pan & Fs - Aegintro & Fo-Ansocan & F10. Linhe \\
\hline ALT $T=$ Termina pause & ALTO $=$ DOS & ALTH $=$ Auda & Ese $=$ Funpos & \\
\hline
\end{tabular}

\section{FIGURA II}

BIBLO2.FIC

DEFINIÇÁO DE FICHA

Item a escolber:

Escollha o item ou use as funçóes

RESUMO

!TEX

\begin{tabular}{|c|c|c|c|c|}
\hline Fi-Inicia & F2. Roves & F3 Arquiva & Fa.Monv & F5-Programa \\
\hline F6 - Gavets & F7 - Pouta & Fe- Regatro & F9. Aseocis & F10-Linns \\
\hline ALT $T=$ Termuna pavea & $A L T D=D O S$ & ALTTH = Apusa & Ekc = Funçbes & \\
\hline
\end{tabular}




\section{CONSIDERAÇŌES FINAIS}

Apesar da informática ter sido considerada, no senso comum, como instrumento de alta complexidade, ficou evidente no presente trabalho, que esta concepção não é verdadeira, pois seus autores, que possuem conhecimentos heterogêneos sobre informática, foram capazes de construir, em curto espaço de tempo, bases de dados para facilitar, agilizar e resolver problemas que se apresentam na prática.

A base de dados AISM permite manusear grande número de informaçöes para o atendimento da mulher, tendo em tempo hábil informaçōes específicas únicas e/ou coletivas além de dados estatísticos.

A base de dados EDUC favorece o acompanhamento da vida funcional dos funcionários, contendo além da avaliaçâo outras informaçōes que podem facilitar o remanejamento e elaboraçāo de escalas de serviço, férias, entre outros.

A base de dados BIBL facilita a elaboração de trabalhos científicos, por conter referência bibliográfica, palavras-chaves e texto contendo resumo referente a cada artigo.

Essas bases de dados reforçam a idéia de que o emprego desta tecnologia constitui-se numa ferramenta que a enfermagem pode utilizar como instrumento facilitador de suas atividades administrativas, assistenciais, de ensino e de pesquisa.

Considerando o exposto, sugere-se que outros estudos sejam realizados para aprofundar questōes relativas à aplicabilidade da informática na enfermagem, o que poderá subsidiar as escolas ao adotarem esta tecnologia em suas atividades didáticas, já que os serviços de saúde estāo caminhando, rapidamente, para a informatização e o enfermeiro necessita estar atualizado para acompanhá-la.

YOSHIOCA, M. R. et al Development of data bases for use in nurving. Rev. Eac. Ent. USP,v.28, a.1,p. 27.40, Apr., 1994.

The purpose of this study is to discuss the utilization of informatics in nursing rsing and the construction of computerized data bases by nursing professionals that have no ave no previous experience with computers. Three data bases developed during the pos gra-os gra duate nursing course are described.

UNITERMS: Data Bases, Informatic.

\section{REFERÊNCIAS BIBLIOGRÁFICAS}

01. BANCO de dados bibliográficos da USP. São Paulo, Sistema Integrado de Biblioteca USP. 1992. (Informes,01) 
02. BEMMERL, J.H.Van. Computer assisted care in nursing - computers at the bedside. Comput.Nurs., v.5, n.4, p. 132.9, 1987.

03. BLOOM,K.C. et al Development of expert system prototype to generate nursing care based on nursing diagnoses. Comput.Nurs.,v.5, n.4, p. 140.5, 1987.

04. OHM, G.M. et al Informática médica: um guia prático. São Paulo, Atheneu, 1989.

05. COLEMAN, E.A. et al The role of centralized computer services in faculty evaluation. Comput.Nurs., v.4., n.6, p.259-64, 1986.

06. GUIMARĀES, S.M. et al Elaboração de software para a prescriçāo de enfermagem na sala de recuperação pós-aneotécica do Hoopital dao Clínicas de Porto-Alegre. Enfoque, v.18, n.1, p.11.6, 1990.

07. JOHANTGEN, M.E.; PARRINELLO, K. Microcomputers: turning the database into unit management information. Nure.Manage, v.18, n.2, p. 30-4,36,38, 1987.

08. KOSIDLAK, J.G.; KERPELMAN, K.B. Managing comunity health nuroing. Comput.Nur., v.5, n.5, p. 175-80, 1987.

09. LABORDE, J.M. Computer graphics: a tol for decision making in nursing. ComputNurs., v.7, n.1, p. 15-20, 1989.

10. LIA-HOAGBERG, B. et al the computerized information system. MCN., v.12, n.1, p. 11-8, 1987.

11. MAC ALINDON, M.N. et al Computer software for nursing; the advances of a Hospital University Liason. Comput.Nurs., v.4, n.1, p.17-26, 1986.

12. MARIN, H. de F. Aquisiçäo de conhecimento e desenvolvimento de um prototipo de sistema eopecialinta em aepistencia pre-natal no atendimento de gestantes normaie. São Paulo, 1991. 147p. Dissertaçāo(Mestrado)-Departamento de Enfermagem, Escola Paulista de Medicina.

13. ROCHA, M.T. et al Base de dadoa bibliogrffica informatizada sobre informatica e enfermagem 1986/1889. São Paulo, Escola de Enfermagem da Universidade de São Paulo, 1992.

14. ROCHA, M.T. Construçāo de uma base de dados particular informatizada. Rov.Paul.Ent., v.11, n.2, p.64-8, 1992.

15.

Roteiro para o uso de fichário eletrónico Kardic. Jundiaí, Medicina Computadorizada, 1988.

16. SILVA,C.M. Informatizagioo de tarefas adminintrativo burocraticas de enfer magem rolncionadas com o proparo para cirurgias eletivass uma proposta. São Paulo, 1991. 89p. Dissertaçäo(Meotrado)-Escola de Enfermagem, Universidade de São Paulo.

17. SPECTOR, A.F. Using computer in administering a continuing education program. J.Contin.Educ.Nurs. v.18, n.4, p. 141-2, 1987.

18. SPON, D.M.; SPONSELLER, M.J. Computers are nursing: the report card. Today's OR Nurse, v.10, n.9, p.26.30, 1988.

19. TEIXEIRA, E. Informńtica na enfermagem. Enfoque, v.15, n.3, p. 60-1, 1987.

20. THEOTO, M. et al Prontuário informatizado num serviço ambulatorial especializado em banseniase. Ent.Cient. n.4, p. 5-9, 1991.

21. YOSHIOCA, M.R. Informática: nova ferramenta no trabalho da enfermagem. In: ENCONTRO INTERAMERICANO DE INFORMÁTICA EM ENFERMAGEM, 1, São Paulo, 1991. Anain, Sāo Paulo, Sociedade Brasileira de Informática em Saúde, 1991, p.126 